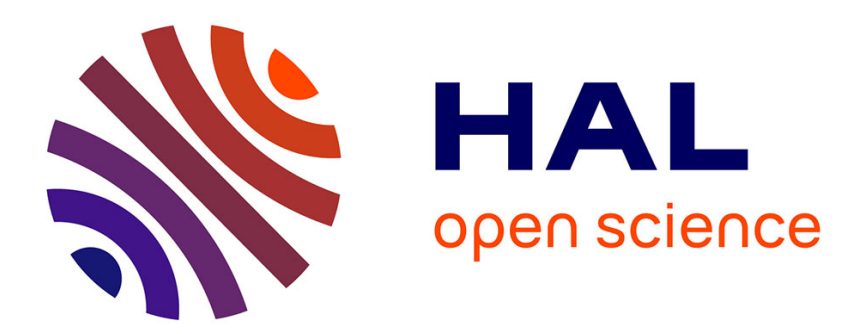

\title{
La conquête des hydrocarbures en Sibérie occidentale, le modèle centre-périphérie revisité
}

\author{
Yvette Marchand-Vaguet
}

\section{To cite this version:}

Yvette Marchand-Vaguet. La conquête des hydrocarbures en Sibérie occidentale, le modèle centrepériphérie revisité. Espace Géographique, 2005, 34 (2), 10.3917/eg.342.0145 halshs-01779818v2

\section{HAL Id: halshs-01779818 \\ https://shs.hal.science/halshs-01779818v2}

Submitted on 22 Jun 2018

HAL is a multi-disciplinary open access archive for the deposit and dissemination of scientific research documents, whether they are published or not. The documents may come from teaching and research institutions in France or abroad, or from public or private research centers.
L'archive ouverte pluridisciplinaire HAL, est destinée au dépôt et à la diffusion de documents scientifiques de niveau recherche, publiés ou non, émanant des établissements d'enseignement et de recherche français ou étrangers, des laboratoires publics ou privés. 


\section{La conquête des hydrocarbures en Sibérie occidentale, le modèle centre-périphérie revisité}

\section{Yvette Marchand-Vaguet}

UMR IDEES 6063, département de géographie, Université de Rouen, 76821 Mont-Saint-Aignan Cedex, yvette.vaguet@univ-rouen.fr

RÉSUMÉ.- La Sibérie occidentale présenteuncas manifeste de changement spatial encore en cours. La région a en effet connu une bifurcation avec l'exploitation de ses hydrocarbures qui a propulsé laRussie au rang des premiers producteurs mondiaux. La transition territoriale met en mouvement des structures spatiales régionales, nationales et internationales. Dans une approche systémique, il est intéressant de lire les multiples temporalités de celles-ci. L'opposition entre le centre moscovite et la périphérie sibérienne perdure mais elle est en plein renouvellement, montrant par-là son caractère fortement résilient.

La Sibérie occidentale demeure une périphérieexploitée. Cependant, elleest de plus en plus intégrée. De sorte qu'il y a diffusion du développement du centre national vers la Sibérie occidentale. Parallèlement, on observe une hétérogénéité croissante de la périphérie sibérienne avec des disparités entre les pôles secondaires et le reste du territoire de la Sibérie.

\begin{abstract}
The epic of hydrocarbon development in Western Siberia, a fresh look at the core-periphery model.Western Siberiais not often studied in geography. However it is extremely interesting as a full-scale laboratory of a case of spatial change in progress since its oil and gas resources have been developed. The region has made Russia one of the leading oil and gas producing countries and its economic function is now more industry-oriented. The territorial transition implies multi-temporalvariations of spatial structures at regional, national and international scale. The core-periphery model is under challenge, but still shows strong resilience. Western Siberia, as a periphery, is becoming increasingly integrated. The spread of development is the main process occurring between the national core and western Siberia. Meanwhile, Siberia as a whole is less and less a homogeneous periphery. The backwash process dominates, and the gap between secondarycores and the rest of Siberia is widening.
\end{abstract}

ENERGY, RUSSIA, SPATIAL CHANGE, TERRITORIAL TRANSITION, WEST SIBERIA a Sibérie occidentale a connu L une bifurcation via un changement définitif de sa fonction économique depuis le développement de l'industrie extractive des hydrocarbures, il y a environ 40 ans. La constante augmentation de la consommation d'énergie mondiale justifie la quête effrénée de ressources, et l'exploitation de cellesci est de nature à imposer des bouleversements aux régions extractrices. Du temps du roi charbon, les pays noirs ont illustré une transition territoriale fonctionnelle. Aujourd'hui, les hydrocarbures dominent le marché mondial de l'énergie. Malgré la perte, depuis la dislocation du bloc soviétique, de la majeure partie du piémont caucasien et de la dépression aralo-caspienne riches en hydrocarbures, la Russie conserve deux grandes régions de gisements situées de part et d'autre de la 
chaîne montagneuse de l'Oural: la zone Oural-Volga surnommée le «second Bakou » et le bassin de l'Ob en Sibérie occidentale. Ce dernier éclairera notre propos dont l'objectifest d'illustrer le cas d'un changement spatial en action aujourd'hui, et ceci audelà de la monographie de la Sibérie occidentale, car cette région est emblématique des transformations spatiales intra- et interrégionales.

À l'époque de l'épopée des hydrocarbures en Sibérie occidentale, la littérature soviétique relate surtout le boom économique régional et tend à le transcender. Les publications, contrôlées par le PCUS, concernent essentiellement l'économie, la géologie et les réussites technologiques. Même les rares contributions des anthropologues de l'époque vantent la conquête et décrivent les avantages pour les populations autochtones. C'est seulement à partir des années 1990, avec la fin de la censure, que les dénonciations du saccage de l'environnement et le cri des peuples du Nord se font entendre (Golovnev, 1992; Kaliakine, 1992). Aujourd'hui, une réflexion de fond sur les bouleversements géographiques se fait encore attendre de ceux qui détiennent les bases de données. Ailleurs, les études ont été limitées en raison d'une part d'une collaboration délicate face à une posture anti-occidentale du monde communiste et, d'autre part, de la difficulté à collecter des données statistiques fiables. La littérature occidentale est, de ce fait, souvent généralisée à l'ensemble de la Russie (Radvanyi, 2000; Brunet et al. 1995; Kempton et Clark, 2002). Les études régionales sont rares et concernent soit de vastes sous-systèmes spatiaux hétérogènes tels le Nord ou la Sibérie et l'Extrême-Orient soit, à une échelle plus fine, l'une des deux capitales du pays. Sur le plan thématique, les contributions occidentales abordent la Sibérie occidentale, entre autres espaces, en traitant des sujets comme les désastres écologiques (Pearce, 1993; Sagers, 1994) ou le secteur économique du pétrole et du gaz (Sagers, 1995, 1996, 2001 ; Von Hirschhause et Engerer, 1998).

Il s'agit ici de faire, à la lumière du changement spatial de cette région, une lecture de la dynamique temporelle du modèle centre-périphérie. D.R. Kempton et T.D. Clark (2002) ont récemment fait une synthèse sur les recompositions politiques dans le cadre de ce modèle sur l'ensemble de l'ex-URSS. Ils discutent comment chaque région se positionne par rapport au centre moscovite, fait sa lecture de l'ouverture et met en œuvre la transition politico-économique. Nous nous plaçons ici à l'échelle de la Sibérie occidentale, une périphérie dont les rapports de forces économiques avec les centres régionaux et nationaux sont en mouvement. Le modèle centre-périphérie oppose alors la Russie européenne et la Sibérie occidentale, ce qui pose comme postulat, a) une dichotomie entre ces deux espaces en termes de peuplement, de richesses et de pouvoir, et b) une homogénéité et une cohérence au sein de chacun de ces deux ensembles. Il faut, dans un premier temps, revenir sur la bifurcation de la Sibérie occidentale, afin de relever en particulier les différentes inscriptions de la transition économique dans l'espace régional. Par la suite, les implications de cette bifurcation sont à discuter à l'échelle des systèmes spatiaux englobants (Sibérie, Russie, Monde). Les évolutions des rapports de la Sibérie occidentale avec le centre moscovite peuvent être abordées notamment dans le cadre du système-Monde. Enfin, la cohérence de la Sibérie, mais aussi de la Sibérie occidentale, est à discuter. Abordant la question du temps, nous nous attacherons, à chaque fois, à mettre en évidence l'induration de certaines structures spatiales, leurs éventuels dysfonctionnements actuels, et l'émergence de nouvelles structures. 


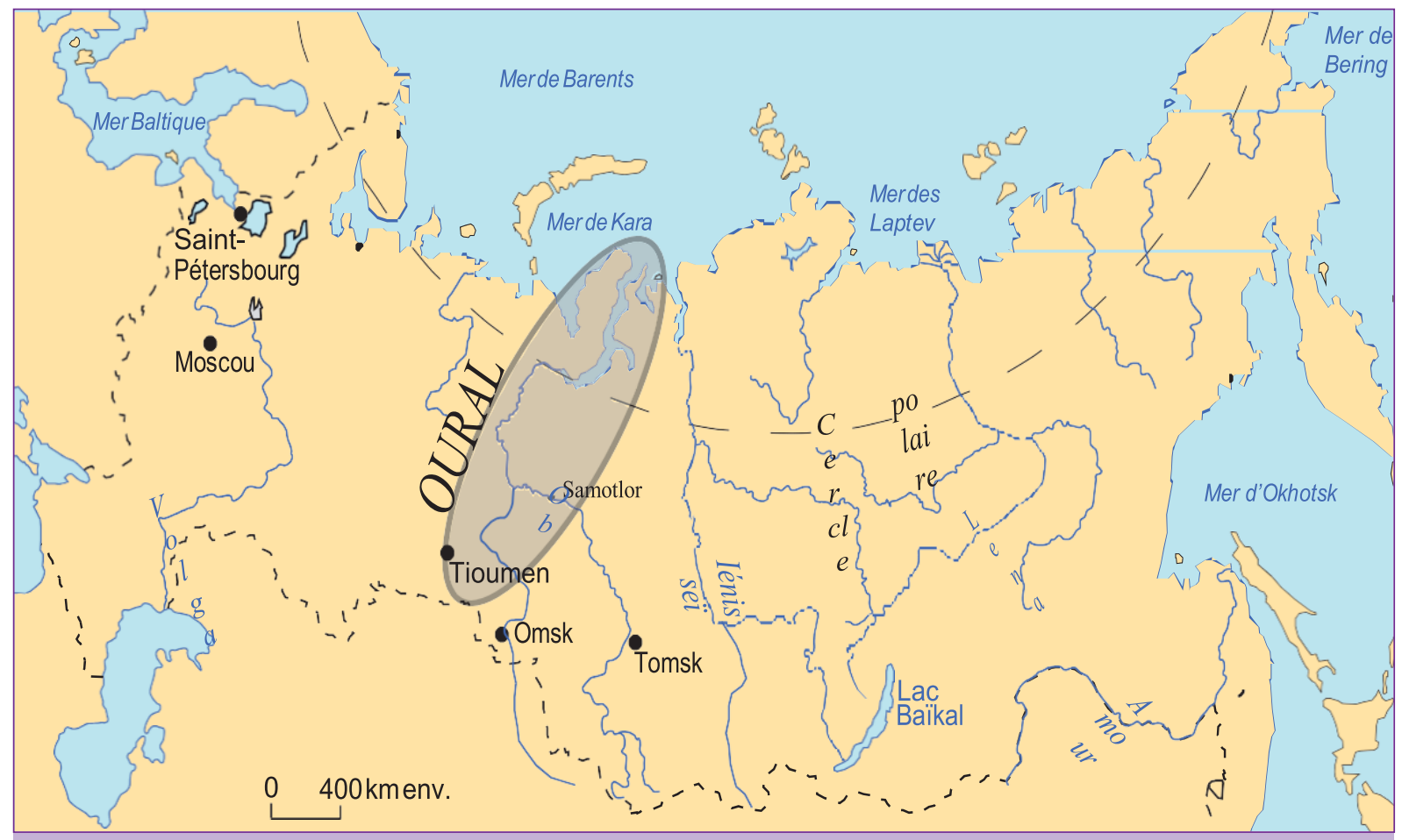

Fig. 1/ Carte de localisation

\section{Retour sur la bifurcation et la transition territoriale}

La référence à des «transitions» suppose qu'en divers lieux doive se répéter la même séquence de phases analogues. Le modèle de la transition démographique illustre cette vision d'une succession de phases liant passé, présent et futur. Il en va de même lorsque l'on parle du modèle classique de développement industriel comportant implicitement le passage de l'artisanat au textile, puis à l'industrie lourde et enfin aux activités de haute technologie. Les références à des «bifurcations » et « événements spatiaux» impliquent une modification durable des composantes et des structures de l'espace dans un laps de temps relativement court (Elissalde, 2000 ; Lepetit et Pumain, 1993 ; Ozouf-Marignier et Verdier, 2000). L'espace géographique est alors dynamique face aux possibilités du présent, toujours susceptible de bifurquer. La bifurcation n'est possible que s'il y a instabilité du système spatial relevant de discordances internes ou de l'occurrence d'une perturbation de grande ampleur qui a, dans ce cas, toutes les chances d'être externe (Elissalde, 2000 ; EPEES, 2000). Elle peut revêtir différentes formes dont celle qui nous intéresse ici : le changement radical de la fonction économique.

Le développement soudain et à grande échelle de l'industrie extractive des hydrocarbures en Sibérie occidentale constitue une bifurcation pour cette région où sont renfermées de gigantesques réserves d'hydrocarbures, les plus grandes du monde pour le gaz. C'est avec l'exploitation du gisement pétrolier de Samotlor (fig. 1) en 1964 que commence la transition économique, avec le passage de la primauté du secteur primaire à celle du secteur secondaire. L'aventure a commencé par une véritable épopée de l'or noir d'abord, puis celle du gaz dans ces rudes contrées septentrionales. 
1. Oblast, okroug et kraï sont des unités administratives qui peuvent être traduites respectivement par région, district et territoire.

Toutefois, nous conserverons les termes russes afin d'éviter toute ambiguïté.

2. La Sibérie occidentale est plus vaste géographiquement puisqu'elle comprend aussi les oblasts de Tomsk, Novosibirsk et Omsk, plusla République et le kraï de l'Altaï. Cependant, ces unités administratives méridionales ne sont pas directement concernées par la «bifurcation» des hydrocarbures.
Ceci concerne plus précisément la province de Tioumen qui se compose de l'oblast ${ }^{1}$ de Tioumen et des deux okroug autonomes de Khantys-Mansis au nord et de IamaloNenets, à l'extrême nord bordant l'océan Arctique (fig. 1). Nous limiterons ici la Sibérie occidentale à la seule province de Tioumen, c'est-à-dire un espace grand comme 2,6 fois la France ${ }^{2}$.

À partir d'une orientation agricole, et plutôt chasse-cueillette-élevage dans les deux okroug, soit dans la majorité du territoire, la région connaît une transition vers l'activité extractive. L'effacement de la fonction « agricole » au profit de la fonction industrielle s'inscrit dans l'espace régional par un changement à $90^{\circ}$ des axes de vitalité (fig. 2). Tandis que le transsibérien concentrait les forces vives dans la partie méridionale, l'exploitation des hydrocarbures invente un nouvel axe de premier ordre vers le nord, une percée vers l'océan Arctique. L'histoire des bassins gaziers durant les trente dernières années en Sibérie occidentale illustre bien cette progression vers le nord, dans des milieux toujours plus rudes (Seligman, 1998). En 1966, le premier gazoduc est mis en fonctionnement au sud de Beloiarskii, dans la plaine de l'Ob, dans de rudes conditions (amplitude thermique annuelle de $80{ }^{\circ} \mathrm{C}$, marécages, per- gélisol discontinu et couche active de $80 \mathrm{~cm}$ ). Plus au nord, c'est en 1972 que l'exploitation du bassin de Medvéjié débutait. Dans cette zone, les processus cryogé- niques sont très répandus, particulièrement dans les nombreux marais. En 1978, le premier gazoduc était posé dans le bassin d'Ourengoï où le pergélisol encore dis- continu et parfois épais de $400 \mathrm{~m}$, peut contenir jusqu'à $50 \%$ de glace. Ce bassin, à lui seul, produit actuellement plus que tous les pays européens réunis et Novyi- Ourengoï est une des villes les plus peuplées du Nord de la Russie (98 500 habi- tants). En 1986, l'ouverture d'un nouveau pipeline inaugurait le bassin de Iambourg dans la toundra de la péninsule de Tazovskii, avec un pergélisol continu, épais de 400 à $560 \mathrm{~m}$ et à haute teneur en glace (80-90 \%). En 2000, le plus grand gazoduc trans- continental a été ouvert entre la péninsule du Iamal et l'Europe occidentale. Aujourd'hui des chantiers qui comprennent des traversées de l'espace maritime arc- tique sont en cours.

Cette transition territoriale s'accompagne d'un gâchis environnemental effroyable. Chaque kilomètre supplémentaire en milieu extrême a un coût environnemental élevé. Or, nulle part ailleurs l'exploitation des hydrocarbures à si vaste échelle n'a eu lieu à aussi vive allure, avec autant de gaspillage et si peu de considération pour l'environnement et les populations locales (Marchand, 1999). Les infrastructures sont soumises à de rudes conditions, augmentant d'autant l'aléa que représente la possible rupture (Marchand et Rees, 1999). En outre, la vulnérabilité des écosystèmes croît avec la latitude et les temps de réhabilitation après perturbation anthropique s'allongent, essentiellement en raison de l'activité micro-bactérienne qui est réduite par le froid. Par ailleurs, il faut signaler que le matériel était d'emblée obsolète et le personnel, allochtone et peu qualifié, était moins sensible à l'environnement qu'aux encouragements à dépasser les prévisions du Plan. Dans ces conditions les premiers accidents ont rapidement eu lieu. Pour le pétrole, les pertes dues aux fuites sont évaluées entre 15 et 30 millions de tonnes par an soit 5 à $10 \%$ de la production nationale. Le lac Samotlor, campé dans un site naturel exceptionnel, a donné son nom au gisement pétrolier proche qui est actuellement parmi les dix plus importants du monde. Il illustre tristement ce saccage : « À présent, ce lac est mort. On n’y trouve rien d'autre que du pétrole...» (Kaliakine, 1992). Il est intéressant de noter ici que la 

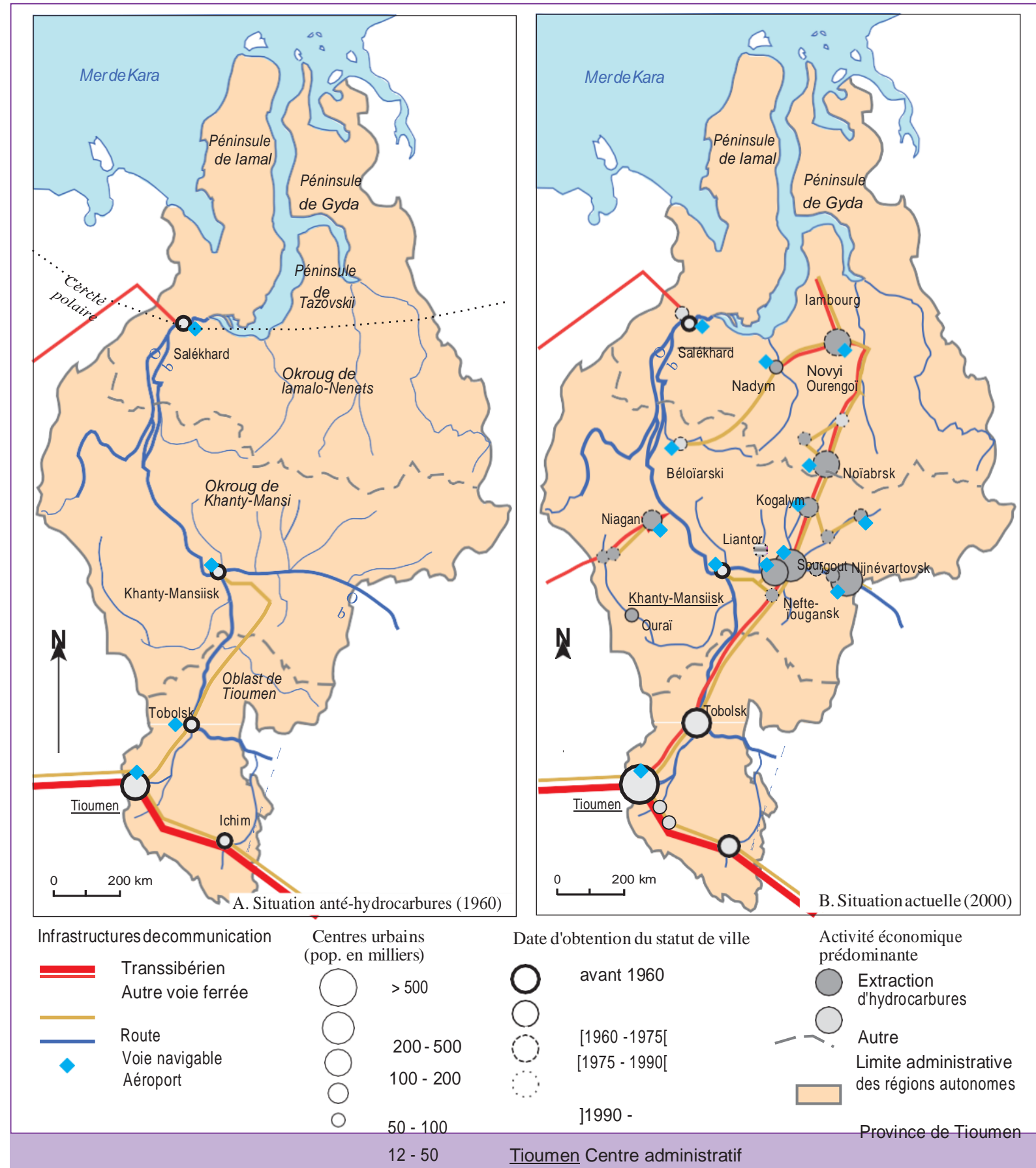

Fig. 2/ Inscription de la transition économique dans l'espace ouest-sibérien

A. Situation pré-transitionnelle, espace structuré par le transsibérien et les voies d'eau. B. Situation actuelle, espace structuré par les voies ferrées, la route et les aéroports.

langue russe désigne par dobitcha, la «proie», le «butin» et l'«extraction»; ainsi dobitcha neft désigne tout à la fois l'extraction pétrolière et le butin pétrolier. Le sac-

VAGUET]-MARChAnD, Y. 2005. La conquête des hydrocarbures en Sibérie occidentale, le modèle centre-périphérie revisité. L'Espace géographique, 2, 145-159. 
cage de la nature est bien réel partout comme le traduit le triste titre de l'article de 
3. « Contamination pétrolièred'Usinsk: catastrophe environnementale ou routine ». L'accident d'Usinsk (Rép. de Komi) s'est produit en septembre 1994. Suite à la rupture d'un oléoduc, 65000 tonnes depétrole brut se sont répandues dans la taïga, soit deux fois plus quelors de la catastrophe de l'Exxon Valdez (Alaska, 1989), et six fois plus que celle de l'Erika (France, 2000).
Vilchek et Tishkov (1995): Usinsk oil spill: environmental catastrophe or routine event ${ }^{3}$. Cette exploitation extensive et prédatrice laisse de moins en moins de place au système régional traditionnel fondé, surtout au Nord, sur un peuplement de nomades vivant de l'élevage, de la cueillette et de la chasse (Golovnev, 1992).

Cette transition territoriale s'accompagne aussi d'un bouleversement du peuplement (Zaitseva, 2002). La population de la province de Tioumen a triplé, passant de 1,1 million à 3,2 millions entre 1959 et 1979 . Ce peuplement rapide a bouleversé les équilibres démographiques régionaux tant numériquement que qualitativement (fig. 2 et 3). Entre les recensements de la population de 1959 et de 1969, l'okroug de Khantys-Mansis, avec celui de la Tchoukotka, a présenté la croissance urbaine la plus forte en Russie. Sur la période 1969-1989, ce sont les deux okroug de la province qui ont présenté les augmentations de la population urbaine les plus fortes de toute la Russie (Brunet et al., 1995). Les Nenets et les Khantes, deux groupes de nomades autochtones, constituaient jadis l'essentiel de la population des deux okroug. Les premiers, les plus septentrionaux, peuplent la toundra et vivent de l'élevage du renne complété par la chasse et la pêche. Les seconds peuplent la taïga du bassin moyen de l'Ob et leur économie est fondée sur la pêche, en association avec la chasse et la cueillette. Les deux okroug étaient alors fortement structurés par les voies d'eau et faiblement peuplés, ne rassemblant que $17 \%$ de la population de la province en 1959. La société moderne sédentaire était à l'époque cantonnée au Sud, dans l'oblast de Tioumen, structuré par le transsibérien. C'est dans cet espace méridional que $83 \%$ de la population de la province se concentrait en 1959. Une des conséquences démographiques majeures des trente années de conquête est l'inversion du poids démographique des unités administratives de la province: en 1989, l'oblast au Sud ne comptait plus que $43 \%$ de la population de la province. Parallèlement, les liaisons ferroviaires, routières et aériennes s'imposaient comme voies de communication structurantes.

Le peuplement inhérent à la conquête du Nord est de type urbain. Ainsi, des

28 villes de la province, 23 ont été créées depuis 1960, dont 21 dans les deux okroug (fig. 2). Au total, 9 seulement ne sont pas directement liées à l'extraction des hydrocarbures. Les villes s'appuient les unes aux autres sur le plan logistique afin de progresser vers le nord, d'où le terme de base arrière (Gavrilova, 1997; Radvanyi, 2000). La figure 3 montre la cohabitation, aujourd'hui, de deux systèmes spatiaux tels que le définissent $\mathrm{Ph}$. et $\mathrm{G}$. Pinchemel (1994), un «système à double solidarité fonctionnelle et formelle» émanant d'une société car «il n'y a pas de société sans spatialité, d'organisation sociale sans une mise en espace qui lui corresponde ». Le premier système spatial en Sibérie occidentale est fondé sur une ruralité, une activité agricole et un mode de vie nomade. L'autre, plus récent, est fondé sur une activité industrielle avec une population urbaine et sédentaire quoique très fluctuante. Tandis que le premier comporte des parcours de transhumance, le second se compose du réseau de villes nouvelles. La cohabitation est bien difficile comme on l'imagine aisément, cependant il serait trop simpliste de réduire la difficulté à la seule concurrence pour l'espace. Certes, celle-ci est de nature à susciter des tensions, mais elle ne doit pas masquer les discordances qui sont apparues en termes de temporalité et de complexité. Le premier système spatial, le plus ancien, se caractérise par une quasi-autarcie et le rythme des transhumances saisonnières nord-sud. Le second système, plus moderne, est ancré dans le système-Monde et évolue au rythme des fluctuations du marché mondial de l'énergie. Que le prix du baril de pétrole chute et les projets de villes plus au nord 
seraient ajournés et les villes actuelles seraient menacées de fermeture. Ainsi, à la concurrence spatiale et fonctionnelle s'ajoute la difficulté de faire cohabiter dans le même espace régional des systèmes spatiaux qui ont des temporalités et des degrés d'ouverture, et donc de complexité, très différents. Par ailleurs, la progression du second système vers le Nord ne doit pas laisser entendre que le Sud est le point de départ de cette conquête. Au contraire, les apports extérieurs à la région sont prépondérants: les plans des villes nouvelles ont été établis à Saint-Pétersbourg, les productions planifiées à Moscou, les nouveaux urbains le plus souvent ne sont même pas sibériens, etc. Les richesses naturelles de la région ont propulsé le pays au rang des premiers pays producteurs d'hydrocarbures et les interactions entre la région et l'espace central se sont intensifiées. Dans le même temps, la nature de celles-ci a été affectée lors de la bifurcation. Ainsi, il est intéressant d'aborder les conséquences sur le modèle centre russe-périphérie ouest-sibérienne.

\section{Variations russes des rapports centre-périphérie}

La consommation mondiale d'énergie par habitant n'a cessé de croître, s'affirmant comme un fait de société qui accompagne chaque révolution sociétale. Elle a doublé lors du passage de la société agricole primitive à la société agricole avancée, triplé lors du passage à la société industrielle et encore triplé lors de l'avènement d'une société technique ${ }^{4}$. La consommation d'énergie devrait encore augmenter d'ici à 2020 et l'hégémonie du pétrole et du gaz, apparue durant les dernières décennies, devrait se renforcer par rappor t aux autres types d'énergie (Maurice, 2001). Or, raisonner en termes de hausse de la consommation énergétique renvoie inévitablement aux croissances démographique et économique auxquelles elle est corrélée positivement. L'Europe prévoit d'augmenter de $2,8 \%$ sa consommation de gaz, se situant ainsi dans la moyenne mondiale, tandis que l'Asie de l'Est et l'Asie du Sud vont respectivement augmenter de $5,2 \%$ et $6,6 \%$. On voit dès lors apparaître les marchés de demain. De fait, les pays de l'Organisation de coopération et de développement économique (OCDE) verront leur consommation relative passer de $54 \%$ de la production mondiale d'énergie primaire à $44 \%$ en 2020, soit
4. Consommation mondiale en TWh : 1,0 (1890), 1,6 (1910), 2,3(1930), 3,3(1950), 8,4 (1970), 13,7 (1990) in Mérenne-Schoumaker, 1997.

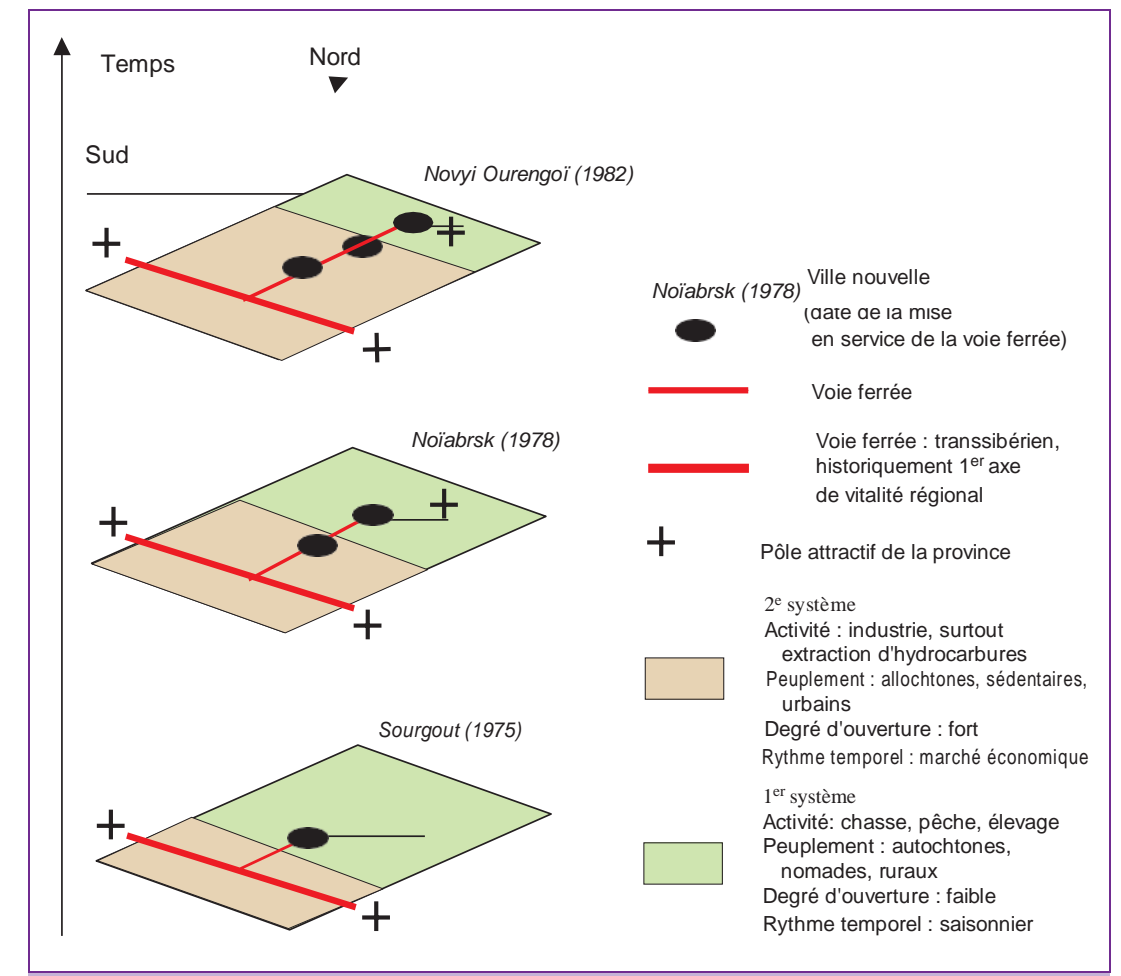

Fig.3/ Systèmes spatiaux et temps au sein de la province de Tioumen 
5. $+139 \%$ pour le Moyen-Orient etl'OPEP, $+29 \%$ pour la Russie et $-15 \%$ pour l'Amérique entre 1997 et2020 (Maurice, 2001).

6. Abréviations usuelles d'unités : tep $=$ tonnes équivalent pétrole; $\mathrm{Mt}=$ million de tonnes ; $\mathrm{Gm}^{3}=$ milliard de $\mathrm{m}^{3}$. un retrait relatif au profit de l'Asie. Cette situation augure une compétition accrue entre les centres de consommation et une convoitise exacerbée pour les centres d'extraction, dont la Sibérie occidentale.

Actuellement, les flux des hydrocarbures font apparaître comme centripètes les trois pôles de la Triade (Europe, Amérique du Nord et Japon) qui consomment donc bien plus qu'ils ne produisent. Les principaux flux internationaux de gaz proviennent de la Russie qui est de loin le premier pays producteur de gaz avec, en 2003, 22,4\% de la production mondiale, devant les États-Unis $(19,9 \%)$ et le Canada $(6,7 \%)$. Cet avantage devrait se maintenir. Quant aux flux internationaux récents de pétrole, ils montrent une baisse de l'hégémonie du Proche-Orient, car la Russie est le deuxième pays producteur $(11,3 \%)$, derrière l'Arabie Saoudite (12,7\%) et les États-Unis $(9,4$ $\%$ ); elle est devenue le deuxième pays pour l'exportation derrière l'Arabie Saou- dite. Cette position devrait se prolonger au vu des augmentations des productions des grands producteurs 5 . Cependant, la situation actuelle au Moyen-Orient est susceptible d'aider la Russie à augmenter ses capacités d'exportation. En effet, les ÉtatsUnis souhaitent que la part de leurs importations de pétrole en provenance de ce pays passe de 1 à $10 \%$ d'ici 2010. Ce projet prévoit, d'ici 2007, l'aménagement d'un terminal pétrolier dans le port arctique de Mourmansk, et un oléoduc le reliant à la Sibérie occidentale, espace de production.

La figure 4 montre comment les productions de la province de Tioumen ont porté la Russie parmi les acteurs mondiaux incontournables du secteur des hydro- carbures, ce bien si convoité dont la demande ne cesse de croître. Pourtant, lorsque les autorités nationales ont imposé et conduit la bifurcation fonctionnelle de la région, les ambitions n'étaient pas de cette ampleur. Il s'agissait de satisfaire la demande énergétique nationale, soit de l'ex-URSS, dont le déficit s'élevait à 100 millions tep ${ }^{6}$ en 1970, et de pourvoir le pays en devises nécessaires à l'achat de denrées alimentaires et d'équipements industriels (Gavrilova, 1997). Or, à chaque avancée vers le nord, l'exploration dévoile les immenses richesses de la nature et la production explose, passant entre 1965 et 1988 de 1 à $415 \mathrm{Mt}$ pour le pétrole, et de 0,3 à $511 \mathrm{Gm}^{3}$ pour le gaz. Pour la première fois en 1982, la production est inférieure aux prévisions du Plan et de gros efforts sont réalisés pour la rétablir. La rapidité avec laquelle les mesures sont prises malgré la lourdeur de l'appareil politique traduit l'ampleur des enjeux dont la région était déjà devenue l'objet. En 1986, les quotas de production sont de nouveau atteints et seront même dépassés. Cependant, ce second souffle ne dure que trois ans et la région plonge définitivement, à la fin des années 1980, dans une crise, à l'instar du pays tout entier qui entrait dans sa transition économique. L'ensemble de l'appareil de production russe a été touché, y compris le secteur des hydrocarbures. Cependant, au-delà des raisons politiques et du contexte national, la crise se profilait dès le commencement de la conquête. Elle apparaît en effet comme le dénouement prévisible d'un développement fondé sur un modèle prédateur et extensif qui écrème les réservoirs les plus faciles à exploiter et qui les abandonne dès la rencontre des premiers obstacles techniques.

La production de pétrole de la province de Tioumen a atteint son maximum en 1988 (415 Mt) puis a baissé de façon constante et rapide jusqu'en 1996 (204 Mt) ; toutefois, elle connaît une timide reprise depuis 1997 (220 Mt en 2000). La participation de la province à la production nationale et mondiale de pétrole est de l'ordre, respectivement de $70 \%$ et de $6 \%$ (fig. 4). La production de gaz de Tioumen a 

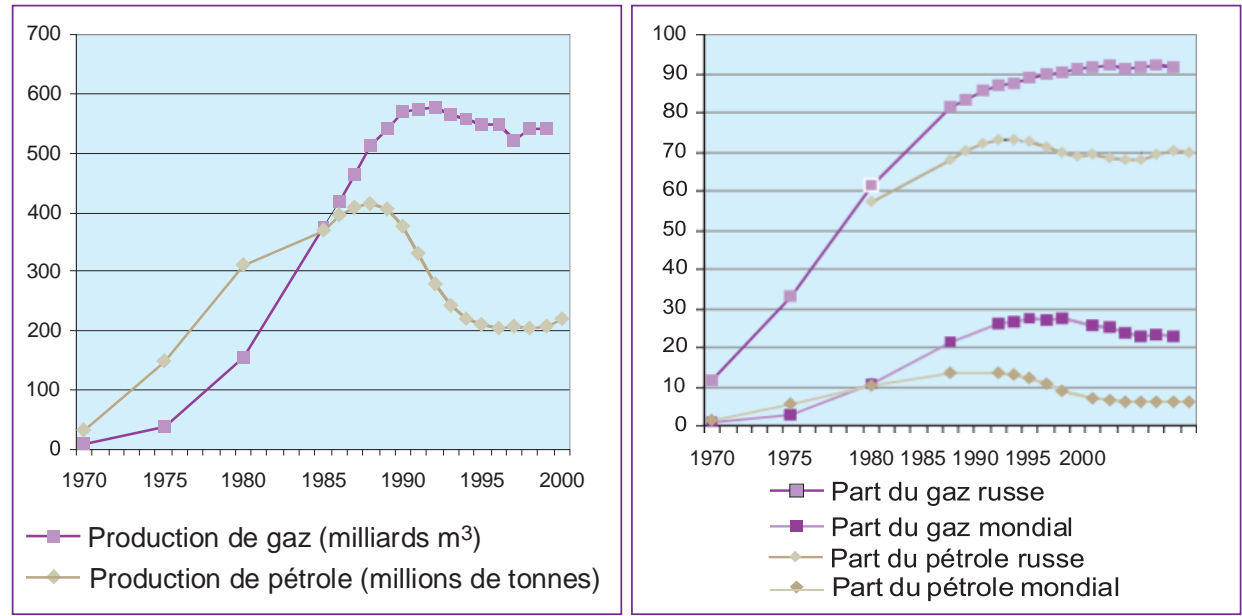

Fig. 4/ Poids énergétique national et mondial de la province de Tioumen(1970-2000)

continué à croître durant la décennie 1980, en dépit de la crise, en réponse à l'avancée du front d'extraction vers le nord qui atteignait les grands gisements gaziers d'Ourengoï (1978-) et de Iambourg (1986-). Elle a atteint son maximum en 1992 (576 $\mathrm{Gm}^{3}$ ) et c'est seulement depuis 1993 qu'elle diminue (523 $\mathrm{Gm}^{3}$ en 1997). La part de la région dans la production nationale est passée de 61 à $92 \%$ entre 1980 et 1994 et stagne depuis. Si la Russie est actuellement l'unique géant du gaz, en pro- duisant quasiment un quart de la production mondiale, c'est donc bien grâce aux champs ouest-sibériens.

Actuellement, l'économie nationale est très dépendante du secteur de l'énergie. Ce dernier est un sérieux outil des stratégies de croissance et de diversification de l'appareil de production national. La part des taxes pétrolières compte pour $40 \%$ dans le budget fédéral de 2001, et Gazprom, la compagnie du gaz, pèse $10 \%$ du PIB national. La Sibérie occidentale est, par voie de conséquence, devenue un atout pour le pays. Néanmoins, cette dépendance signifie aussi une fragilité qu'a soulignée F. Benaroya (2000) en écrivant que « la crise ne serait pas intervenue en août 1998 si le cours du pétrole avait été alors ce qu'il est aujourd'hui». En effet, «les autorités estiment que le budget fédéral perd 1 milliard de dollars chaque fois que le prix du pétrole baisse de 1 dollar» (Nougayrède, 2001).

Cet état de fait nourrit l'hypothèse que les rapports de la région au centre moscovite ont dû évoluer. Afin d'en discuter, il n'est pas inutile de revenir sur la typologie des classes socio-spatiales d'Alain Reynaud (1981). Il soulignait qu'une périphérie ne peut produire massivement sur le long terme pour le centre sans risque d'affaiblissement, voire de disparition, et donc que ni les centres ni les périphéries ne sont figés dans leurs rôles respectifs. Dans notre espace d'étude, l'épopée des hydrocarbures aura servi l'induration du modèle centre-périphérie, lequel s'est néanmoins renouvelé. $\mathrm{Au}$ départ, on peut considérer le centre comme dominant et la Sibérie occidentale comme une périphérie assurément exploitée. Quant à son niveau de domination ou d'intégration, elle était sans doute plus dominée qu'intégrée. Certes des capitaux ont bien été injectés pour le développement industriel de la région; certes des populations ont été encouragées à migrer vers la province, mais ces flux ne s'inscrivaient pas dans 
7. Le coût

de l'aménagement

du port de Mourmansk et la réalisation de l'oléoduc le reliant àlaSibérieoccidentale serait de 3,4 ou 4,5 milliards de dollars selon le tracé de l'oléoduc. le cadre d'une politique d'aménagement harmonieux ni de mise en valeur, mais d'une domination, d'une exploitation aveugle, qui devait laisser la région exsangue. Ainsi, il semble que les rétroactions n'aient jamais été autres que positives, orientées vers le renforcement du centre. Tioumen fut, dès le commencement de l'aventure, destinée à asseoir le pouvoir russe au sein du bloc soviétique et M.H. Mandrillon (1993) évoque ainsi un «saccage de la nature russe au nom du marxisme-léninisme ». Encore aujourd'hui, le pays s'appuie sur la région, comme un allié certain, dans le processus de transition économique, et le personnel rencontré sur place, le plus attaché à ce territoire, déplore de subir des décisions qui viennent toujours de Moscou.

Néanmoins, il ne faut pas nier qu'en dépit du gâchis environnemental et de l'instrumentalisation de la région par l'autorité centrale en matière de politique de développement, le corollaire de cette épopée des hydrocarbures a été le formidable bond économique de la province de Tioumen. Elle a de fait bénéficié d'investissements, exogènes à la région, considérables entre 1964 et 1990 (Logunov, 1999). Aujourd'hui, la Russie doit composer avec les observateurs, partenaires et bailleurs de fonds internationaux dont la pression n'est jamais totalement désintéressée dans une région où se trouvent de grandes réserves mondiales d'hydrocarbures, les plus grandes pour le gaz. L'ouverture du secteur des hydrocarbures aux investisseurs étrangers est nécessaire, vu le prix élevé qu'il faut payer pour le moderniser, et qui ne peut être supporté par les seuls industriels russes et le gouvernement d'un pays en difficulté financière.

Les investissements étrangers en Russie s'élevaient à 200-300 millions de dollars en 1994 et à 1,2 milliard en 1999, mais ils sont redescendus à 441 millions en 2000 (Sagers, 2001). Du reste, la Russie ne parvient pas à être attractive. Toutefois, les investissements étrangers pourraient bien remonter car tous les clients actuels ne boudent pas l'espace qui renferme les plus grandes réserves mondiales d'hydrocarbures, en raison des enjeux de demain face à une concurrence accrue pour l'approvisionnement. Ainsi, les investissements états-uniens pourraient bien augmenter, malgré les réticences de ceux-ci et celles des compagnies pétrolières russes ${ }^{7}$. De son côté, l'Europe, pourtant un créancier important de la Russie, tarde à s'impliquer dans l'assainissement de la crise qui frappe ce territoire sur ses marges. Ainsi la France, qui avait la Russie comme principal fournisseur de gaz, s'est peu à peu tournée vers la Norvège. Le géographe J. Lévy (1997) donne le ton des relations entre l'Europe et la Russie, en intitulant un chapitre de son ouvrage « Le problème Russie », où il évoque « une périphérie promouvable mais non intégrable» ou encore une Russie qui s'emploie à devenir un « partenaire fréquentable». Quoi qu'il en soit, la Sibérie occidentale est la seconde région de Russie, derrière le centre européen, à recevoir les capitaux étrangers (Gicquiau, 1997). D’ores et déjà la pénétration étrangère et l'émergence de nouvelles formes d'organisation ont relancé la produc- tion et la productivité des puits qui avaient considérablement chuté (indice 100 en 1990, 30 en 1999 et 60 en 2000). Les firmes étrangères interviennent, de plus en plus, dans la revalorisation des gisements existants (Pany, 2002). Ceci amoindrit la valorisation de nouveaux champs qu'on réserve peut-être pour le futur, notamment lorsque la législation russe sera plus favorable aux investisseurs étrangers et les prix plus élevés. Sur place, on observe l'amélioration des conditions de travail dont les standards sont rehaussés par la présence des firmes étrangères.

Finalement, les rétroactions négatives, c'est-à-dire des effets positifs pour la périphérie ouest-sibérienne, s'intensifient. Les signes d'intégration se multiplient même si 
les rênes demeurent tenues par des compagnies et entrepreneurs rarement localisés dans la région. Le modèle centre-périphérie a donc évolué au profit de la périphérie qui reste néanmoins dépendante du centre moscovite. La situation correspond, sur le diagramme de M.J. Moseley (1974), à une réduction dans le temps de l'écart en termes de niveau de développement entre le centre et la périphérie. Cependant, cette diffusion ne touche pas l'ensemble de la Sibérie. Il reste maintenant à aborder les conséquences de la bifurcation et du développement de la Sibérie occidentale pour cet espace périphérique russe vu comme un ensemble cohérent.

\section{Variations sibériennes de la périphérie}

L'espace asiatique russe s'est grossièrement développé en «peigne », dont les différentes dents traduisent l'avancée de la colonisation russe depuis le cœur européen vers l'est et le nord. La pénétrante principale ouest-est consiste en un axe ferroviaire très décalé au sud : le transsibérien. Il concentre la majorité des hommes et des activités dans ce qui est appelé la «Sibérie utile» (Brunet, 1996). La navigation sur les grands fleuves au cours sud-nord permet d'assurer des couloirs de communication, telle la liaison Krasnoïarsk-Doudinka sur l'Iénisseï, complétant l'image du peigne. De ce fait, c'est à la croisée du transsibérien et des fleuves que sont localisés les plus grands centres urbains (Omsk sur 1'Irtych, Novossibirsk sur l'Ob, Krasnoïarsk sur l'Iénisseï). Les infrastructures routières et ferroviaires viennent inégalement compléter ce dispositif rudimentaire. Il s'agit le plus souvent de voies sud-nord embryonnaires. L'axe Tioumen-Novyi Ourengoï est le plus étiré vers le nord et, en ce sens, le plus achevé. La liaison Tynda-Iakoutsk, en Extrême-Orient, est achevée par la route mais la voie ferrée s'arrête à Aldan.

Ce dispositif en peigne avait autrefois été rêvé à partir de la voie maritime du nord avec pour nœuds de transport les ports situés aux embouchures des grands fleuves. Sibiriakov, un commerçant du $\mathrm{XIX}^{\mathrm{e}}$ siècle, avait financé deux expéditions, imaginant ainsi importer sur le marché européen le blé et la soie de porc de Sibérie. La crise de 1870 ajourna ses projets et le transsibérien fut ordonné en 1891 (Steber, 1936). Le dispositif aurait pu être une échelle dont les barreaux auraient été les fleuves, routes et voies ferrées, et les montants, le transsibérien au sud et la voie maritime au nord. Cette dernière n'est ouverte toute l'année que depuis 1978, et encore, elle s'arrête à Doudinka. Finalement, l'immensité du territoire et les contraintes du milieu extrême ont conduit à multiplier le recours aux transports aériens. Les liaisons aériennes font dès lors apparaître une structure de relations spatiales indépendante de la trame en peigne, et un lieu situé dans le Grand Nord peut être mieux relié à Moscou qu'à sa capitale régionale en position méridionale. Dans tous les cas, l'aménagement du territoire répond aux exigences de rapporter les richesses sibériennes au centre européen. $\mathrm{Au}$ XVIII ${ }^{\mathrm{e}}$ siècle, le grand scientifique russe Mikhail Lomonosov déclarait : "La Sibérie rendra la Russie riche» mais aujourd'hui, l'éclairage porterait plutôt sur l'autre versant de cette structure spatiale, à savoir que la Russie rend la Sibérie pauvre.

Il n'en demeure pas moins que l'organisation spatiale de l'Asie russe a induit un cloisonnement longitudinal avec une subordination des espaces septentrionaux aux espaces méridionaux. Il s'agit là d'un gradient en termes de peuplement, d'activités économiques et de pouvoir politique, depuis le transsibérien vers le nord : de la Sibérie utile aux espaces «vides». L'organisation des sujets de la Fédération illustre 
parfois cette subordination des okroug autonomes du Nord aux oblasts du Sud (Khanty-Mansiisk et Iamalo-Nenets sont subordonnés à l'oblast de Tioumen, la ville arctique de Norilsk est rattachée à l'oblast de Krasnoïarsk...).

Le modèle centre-périphérie de la Russie présente implicitement la Sibérie comme un tout sous-développé, cohérent et hiérarchisé depuis l'extrémité septentrionale des dents du peigne aux pôles régionaux sur le transsibérien, eux-mêmes subordonnés à l'espace central situé en Europe. Son exploitation a évolué dans le temps, s'adaptant aux conditions nouvelles : bien sûr, à chaque découverte de richesses exploitables selon la demande, mais surtout au gré des évolutions politiques qui ne sont pas minces en Russie. Avec l'Empire d'abord, lequel a mis en place cette structure lors de la colonisation de la Sibérie, puis durant la période communiste et aujourd'hui post-communiste. Comme nous l'avons vu pour la région étudiée, le développement d'un sous-espace sibérien relève de l'autorité centrale et les apports extra-régionaux, à l'initiative du centre, sont souvent prépondérants, par exemple en matière de peuplement (par des détenus autrefois, puis par des pionniers attirés par de hauts salaires).

Dans la province de Tioumen, la systémogénèse induite par la bifurcation a donné lieu à l'émergence de cet espace au sein de l'Asie russe. Cette région était, comme l'ensemble de la Russie asiatique, en état de sous-développement et donc à un niveau bien inférieur au standard de la Russie européenne. C'était une périphérie oubliée, avec un Nord totalement ignoré comparativement au Sud, traversé par le transsibérien. Actuellement, la province est devenue un «Émirat sibérien» et n'a plus grand chose de commun avec les autres régions de Sibérie. Le revenu mensuel moyen des ménages par tête y était, en 1995, le double de celui de l'ensemble de la Russie, et près de 4 fois celui de l'espace asiatique russe (Crosnier, 1997). On assiste là à un processus de polarisation (Moseley, 1974) marqué par un accroissement des disparités. De fait, les écarts interrégionaux au sein de la périphérie se creusent et rendent utopique l'objectif de la charte sibérienne qui est d'obtenir une redéfinition des relations entre le centre et la Sibérie vue comme un tout.

À une échelle encore plus fine, il faut souligner l'apparition de tensions entre l'oblast du Sud et les deux okroug du Nord. Ceux-ci avaient été créés et placés sous la juridiction de l'oblast par Staline, soit bien avant l'exploitation des hydrocarbures. Le conflit concerne les taxes et la redistribution de l'impôt au sein de la province. Rappelons que les unités administratives de la Russie sont divisées entre les «sujets donneurs» qui sont des contributeurs nets au budget fédéral, et les «régions dépressives ». La province de Tioumen, grande région productrice de ressources énergétiques, est une grande donneuse alors que la Sibérie compte une majorité de régions dépressives (Radvanyi, 2000). Toutefois, à bien y regarder, l'oblast qui se voudrait un centre régional dans le sens plein du terme c'est-à-dire pas uniquement politique, n'en a guère l'étoffe car les richesses se situent surtout dans l'okroug de Khanty-Mansi pour le pétrole, et dans l'okroug de Iamalo-Nenets pour le gaz. Quant aux compagnies, leur siège est souvent à Moscou. Aujourd'hui, ce sont les districts septentrionaux qui donnent le plus, comparés au Sud dépourvu de richesses. À cet égard, ils acceptent de plus en plus mal cette dépendance vis-à-vis de ce Sud dont le rôle est limité, notamment, à l'accueil scolaire et universitaire des enfants des salariés de l'industrie extractive et des retraités de ce secteur. C'est ainsi que la constitution de la Fédération de la Russie (1993) a accordé plus d'autonomie aux capitales des okroug (Khanty-Mansiisk 
et Salékhard). Des discordances internes à l'origine de tensions régionales sont apparues et rendent le système instable. Ainsi, il n'est plus certain que la Sibérie occidentale utile soit encore située dans le Sud.

\section{Conclusion}

Finalement, il est indéniable que la Sibérie occidentale a connu une bifurcation dans les années 1960 et l'épopée des hydrocarbures constitue l'assise du changement spatial. Dans un monde où l'énergie est primordiale et sa demande en perpétuelle croissance, la région est devenue stratégique. La systémogénèse a amoindri l'ancienne fonction rurale tandis qu'avait lieu une industrialisation massive et soudaine. Celle-ci est un processus qui se poursuit aujourd'hui sur les rives de l'Arctique lorsque le prix des hydrocarbures permet la rentabilité de leur exploitation en un tel milieu hostile. Inversement, la chute des prix peut provoquer la suspension des chantiers. Par ailleurs, la systémogénèse s'inscrit par une rotation à $90^{\circ}$ des axes de vitalité de la province. En conséquence, la région présente la cohabitation de deux mondes qui se superposent aujourd'hui tandis qu'ils se juxtaposaient encore hier, l'un cantonné au Sud, l'autre au Nord. Or, ceux-ci ont des rythmes, des niveaux d'ouverture et de complexité, très différents. La bifurcation a engendré de nouvelles discordances internes. Elle a débouché notamment sur des conflits qui opposent des Russes européens, autrement dit, dont les enjeux échappent aux populations autochtones. Ces tensions fragilisent ce vaste espace.

Lorsque l'on raisonne à des échelles englobant ce système régional, la transition fonctionnelle de la Sibérie occidentale a eu aussi des conséquences notables. Il n'est plus possible de faire une lecture classique du contraste entre la Russie européenne et la Sibérie, qui reposait d'une part sur le postulat que ces espaces étaient cohérents, et d'autre part sur un gradient sud-nord en Sibérie en termes de peuplement, de richesses et de pouvoir. En disant «Nous voyons la Russie comme jouant un rôle pivot en matière de sécurité énergétique mondiale », le Secrétaire étatsunien à l'énergie, Spencer Abraham, résume la situation actuelle : la région demeure une périphérie exploitée qui n'a guère sa place dans les discussions. Néanmoins, elle est devenue un instrument incontournable pour le développement du pays, l'approvisionnement en hydrocarbures du système-Monde et une alternative non négligeable à la zone OPEP. Les États-Unis n'hésitent pas à y investir tandis que l'Europe se montre encore frileuse. La région voit ainsi les rétroactions négatives se multiplier et elle devient une périphérie exploitée plus intégrée qu'auparavant. De plus, le principe de l'unicité de la périphérie asiatique est remis en cause. Certes, depuis l'époque tsariste, l'Asie russe demeure un puits de richesses au service de l'espace central; cependant, un processus de polarisation des richesses est en cours. À cet égard, la Sibérie occidentale se distingue parmi les mieux «lotis» du pays, véritable «Émirat sibérien».

L'organisation spatiale en peigne se référant aux différenciations des rapports hiérarchiques entre les espaces «vides» du Nord et les espaces «utiles» le long du transsibérien, s'est altérée. Des pôles secondaires sibériens nordiques ont émergé. Le réseau de transport aérien, parce que plus récent et plus souple face aux lourdes contraintes de distance et du milieu naturel, met en évidence ces réorganisations spatiales. Il serait alors intéressant d'étudier la dynamique des pôles régionaux structurants. La province de Tioumen est certainement le meilleur exemple pour discuter des temporalités des figures spatiales. Quel rôle-relais Tioumen joue-t-elle encore aujourd'hui? Dans quelle 


\section{Remerciements}

Cetravail estactuellement soutenu par le ministère français de la Recherche dans le cadre de l'Action Incitative Jeune Chercheur (Projet $n^{\circ} 67007$ ). mesure ce pôle méridional est-il détrôné politiquement par les capitales des okroug, Salékhard et Khanty-Mansiisk, et économiquement par les villes pétrolières et gazières? Ou à l'inverse, dans quelle proportion l'exploitation des hydrocarbures de la province a-t-elle conforté Tioumen comme capitale régionale?

Références

$B_{\text {EnAROYA }} F$. (2000). "La dernière grande crise russe (août 1998). Autopsie et séquelles 》. Géoéconomie, n¹3, p. 11-34.

B RUNET R., REY V. (dir.), (1996). Europes orientales, Russie, Asie centrale. Vol. 10, Géographie universelle. Paris/Montpellier : Belin/Reclus, $480 \mathrm{p}$.

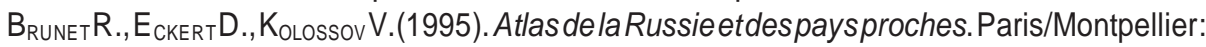
Reclus/La Documentation française, coll. «Dynamiques du territoire », $208 p$.

Cordonnier C. (2000). «La Russie etl'Europe: de la frustrationàl'espoir?» Géoéconomie, n¹3, p. 99-119.

C Rosnier M.A. (1997). " La Sibérie et l'Extrême-Orient russe : déséquilibres et ruptures de croissance ». Courrier des Pays de l'Est, n 422, p. 4-28.

ELISSALDE B. (1999). «Temporalités etcontraste Armor/Arcoat en Bretagne ». Travaux del'Institut de Géographie de Reims, n 101-104, p. 179-200.

E LISSALDE B. (2000). “Géographie, temps et changement spatial ». L'Espace géographique, n 3 , p. 224-236.

EPEES (2000). «Événement spatial ». L’Espace géographique, n 3, p. 193-199.

$F_{A c O N}$ I. (2001). "Quelle Russie en 2001 ? Entretien avec Pierre Verluise ». www.diploweb.com

GavriLovaN.O.(1997). «Le complexe des villes pétrolières et gazières de Sibérie occidentale etsa mise en œuvre (1960-1980) ». Actes de la conférence Espace social, p. 73-84 (en russe).

Golovnev A. (1992). " La Sibérie du Nord-Ouest : une crise éco-culturelle ». In Questions sibériennes, Écologie et culture, IMSECO, p. 7-15.

GICQuiau H. (1997). «L'industrie de la Sibérie et de l'Extrême-Orient russe ». Le Courrier des pays de l'Est, n 422, p. 32-61.

Guellec J. (2000). «La tentation eurasienne de la Russie ». Géoéconomie, n 13, p. 121-136.

K KLIAKINE V. (1992). "Désastre en Sibérie occidentale ». In Questions sibériennes, Écologie et culture, IMSECO, p. 16-19.

$\mathrm{K}_{\text {EMPTON D.R., C }}$ LARK T.D. (2002). Unity or Separation. Center-Periphery Relations in the Former Soviet Union. Londres: Ed. Praeger, $300 \mathrm{p}$.

LePetit B., Pumain D. (1993). Temporalités urbaines. Paris : Anthropos, $316 \mathrm{p}$.

LÉvY J. (1997). Europe, une géographie. Paris : Hachette, 288 p.

Logunov E.V. (1999). Demographic and social consequences of oil and gas development in Siberia. Fairbansk, Alaska : University of Fairbanks, PhD thesis, $264 \mathrm{p}$.

MANDRILlon M.H. (1993). «Russie: rapport à la nature et quête d'identité ». In Bourg D., Les Sentiments de la nature. Paris: La Découverte, coll. «Essais », p. 162-174.

$M_{\text {ARCHAND }}$ Y. (1999). «Industries des hydrocarbures, environnement et télédétection dans le domaine polaire russe ». Revue de Géographie de Lyon, vol. 74, n³ 3. p. 267-274.

MARCHAND $Y ., R_{E E S} G$. (1999). "Remote Sensing and GIS for oil contamination of frozen terrain application ». Proceedings of $10^{\text {th }}$ Int. Conference on Cold Regions Engineering: Putting Research into Practice. Lincoln (NH, USA), 16-19 August, p. 363-373.

MAURICE J. (2001). Prix du pétrole. Rapport du conseil d'Analyse économique. Paris : La Documentation française, $195 \mathrm{p}$.

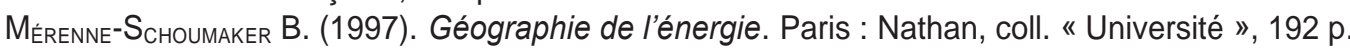


Moseley M.J. (1974). Growth Centres in Spatial Planning. Oxford : Pergamon.

NougayredeN. (2001). «Lafragilecroissance del'économie». LeMonde,3décembre2001, p. 15

OZOuF-M ARIGNIER $_{\text {M., }} V_{\text {ERDIER }}$ N. (2000). « L'événement: un objet historique à emprunter ». L'Espace géographique, $n^{\circ} 3$, p. 218-223.

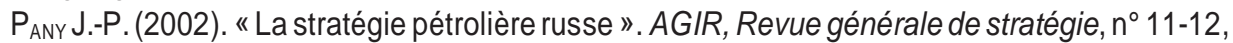
numéro spécial « Stratégie russe pour le ${ }_{x x \mid}{ }^{e}$ siècle », p. 129-143.

$P_{\text {Earce }}$ F. (1993). «The scandal of Siberia ». New Scientist, 27 november, p. 28-33.

Pinchemel Ph., Pinchemel G. (1994). La Face de la Terre. Éléments de géographie. Paris : Colin, coll. «U », $517 \mathrm{p}$.

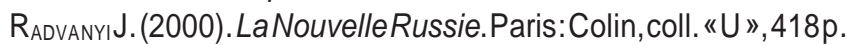

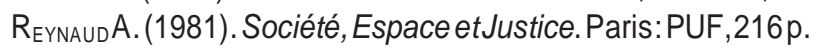

S AGERS M.J. (1994). "Oil spill in Russian Arctic ». Polar Geography and Geology, vol. 18, n², p. 95-102.

S AGERS M. (1995). "The Russian natural gas industry in the mid-1990s ». Post-Soviet Geography and Economics, vol. 36, n 9, p. 521-564.

S AGERS M. (1996). «Russian crude oil production in 1996 ». Post-Soviet Geography and Economics, vol. $37, n^{\circ} 9$, p. 523-587.

S AGERS M. (2001). «Developments in Russian crude oil production in 2000 ». Post-Soviet Geography and Economics, vol. 42, n³, p. 153-201.

Seligman B. (1998). Key factors influencing the reliability of trunk gas pipelines in the West Siberian North. Cambridge: University of Cambridge, PhD thesis, $288 \mathrm{p}$.

Steber C. (1936). La Sibérie et l'extrême-Nord soviétique. Paris : Payot, 245 p.

VILCHEK G.E., TISHкоv A.A. (1995). « Usinsk oil spill : environmental catastrophe or routine event ». In Proceedings of Disturbance and Recovery in Arctic Lands An Ecological Perspective, Crawford.

$\mathrm{V}_{\text {ON }} \mathrm{H}_{\text {IRSCHHAUSE }} \mathrm{C}$., $\mathrm{E}_{\text {NGERER }} \mathrm{H}$. (1998). «Post-sovietgas sector restructuring inthe CIS: a political economy approach ». Energy Policy, vol. 26, n 15, p. 1113-1123.

$Z_{\text {AITSEVA }}$ A.N. (2002). "La population de la province de Tioumen au xx siècle ». Espace Social, vol. $20, n^{\circ} 5-6$, p. 65-70 (en russe). 